\title{
An Extension Theorem for Conditionally Additive Functions and Its Application for the Equality Problem of Quasi-Arithmetic Expressions
}

\author{
Pál Burai
}

\begin{abstract}
The focus of this paper is the equality problem of quasi-arithmetic expressions. This class is a far generalization of the well-known class of quasi-arithmetic means. One of the main tools in the proof is an extension theorem of real homomorphisms from a subset with a very weak structure (dyadically closed set).
\end{abstract}

Mathematics Subject Classification. 39B22, 39B55, 39B82, 39B12.

Keywords. Quasi-arithmetic expressions, extension theorems, regularity, quasi-arithmetic means.

\section{Introduction}

A two place function $M$ is called a quasi-arithmetic mean (see e.g. [1]) if it can be written in the form

$$
M(x, y)=\varphi^{-1}\left(\frac{\varphi(x)+\varphi(y)}{2}\right), \quad x, y \in I,
$$

where $I$ is a non-empty, open interval, $\varphi: I \rightarrow \mathbb{R}$ is a continuous, strictly monotonic function. This class is a close relative of quasi-sums (see e.g. [19]) and it is in the center of research of several authors (see e.g. [8] and the references therein). It contains classical well-known means like the arithmetic mean, geometric mean, harmonic mean and so on. Currently the operator version is also defined and investigated (see e.g. [10]). 
If we consider only the expression (1) itself, then invertibility of $\varphi$ with Jensen convex image seems to be more natural assumption than continuity with strict monotonicity.

The following questions arise: What will be the resulted new class? Is it larger than the class of quasi-arithmetic means? The members of this new class will be means again or not? What are the necessary and sufficient conditions of equality of two members of this class?

The main goal of this paper to answer these questions, especially the last one (equality problem in this class).

The remaining part is organized as follows. In Sect. 2 an extension theorem is proved which is one of the main tools in the proof of the solution of the equality problem. Extension theorems has a quite rich literature because conditional problems concerning functional equations have an important role in this area (see e.g. $[3,7,12,24,25])$.

In Sect. 3 properties of quasi-arithmetic expressions are investigated. It is proved that this class is much larger than the class of quasi-arithmetic means. Examples make the connection between these classes more plausible.

In Sect. 4 the solution of the equality problem is solved in the class of quasi-arithmetic expressions. Such type of questions are vividly examined by several authors (see e.g. [4-6,13-18,20-23]).

At last, in Sect. 5 two open problems are shown concerning the characterization problem of quasi-arithmetic expressions.

\section{An Extension Theorem for Conditionally Additive Functions}

Let $D \subset \mathbb{R}$ be a subset, and

$$
D_{\Delta}:=\left\{(x, y) \in \mathbb{R}^{2} \mid x, y, x+y \in D\right\} .
$$

A function $g: D \rightarrow \mathbb{R}$ is called conditionally additive on $D$ (see [7]) if

$$
g(x)+g(y)=g(x+y), \quad(x, y) \in D_{\Delta} .
$$

A set $D \subset \mathbb{R}$ is dyadically closed if $\frac{x+y}{2} \in D$ for all $x, y \in D$.

The dyadic hull of $D$ is

$$
\operatorname{diad} D:=\left\{x \in \mathbb{R} \mid x=\sum_{i=1}^{n} \delta_{i} d_{i}, \text { for some } n \in \mathbb{N} \text { and } d_{i} \in D\right\} \text {, }
$$

where $\delta_{i}=\frac{m_{i}}{2^{k_{i}}}, m_{i}, k_{i} \in \mathbb{Z}$.

Theorem 1. Assume that a real valued function is defined on a nonempty, dyadically closed subset of the reals, which contains zero. If it is conditionally additive on this set, then it is (not necessarily uniquely) extendible onto the whole real line.

Lemma 1. If $D \subset \mathbb{R}$ is nonempty, dyadically closed set, and it contains zero, then $\operatorname{diad}(D \cap[0, r])=\operatorname{diad} D$ for all positive $r \in D$. 
Proof. Let $d \in \operatorname{diad} D$, then $x=\sum_{i=1}^{n} \delta_{i} d_{i}$ and for and arbitrary $d_{i} \in D$ there exists $j_{i} \in \mathbb{N}$, such that $\operatorname{sign}\left(d_{i}\right) \frac{1}{2^{j_{i}}} d_{i}<r$. So

$$
x=\sum_{i=1}^{n} \delta_{i} d_{i}=\sum_{i=1}^{n} \frac{m_{i}}{2^{k_{i}}} d_{i}=\sum_{i=1}^{n} \underbrace{\frac{\operatorname{sign}\left(d_{i}\right) 2^{j_{i}} m_{i}}{2^{k_{i}}} \operatorname{sign}\left(d_{i}\right) \frac{1}{2^{j_{i}}} d_{i}}_{\in \operatorname{diad}(D \cap[0, r])} .
$$

So, $\operatorname{diad} D \subset \operatorname{diad}(D \cap[0, r])$. The other direction of the inclusion is trivial.

Proof of Theorem 1. If $D$ has a single element, the statement is trivial.

We can assume without losses that $D$ has at least two elements, and at least one is positive, say $0<r \in D$. Because of the previous lemma, it is enough to prove the statement for $D_{r}:=D \cap[0, r]$.

For an arbitrary $x \in \operatorname{diad} D_{r}$ there is a unique integer $n_{x} \in \mathbb{Z}$ such that

$$
n_{x} \frac{r}{2} \leq x<\left(n_{x}+1\right) \frac{r}{2} .
$$

Let $x^{\prime}:=x-n_{x} \frac{r}{2}$. Because of its definition $x^{\prime} \in D_{r}$. Assume that $g: D_{r} \rightarrow \mathbb{R}$ is conditionally additive, and let's define

$$
a: \operatorname{diad} D_{r} \rightarrow \mathbb{R}, \quad a(x):=n_{x} g\left(\frac{r}{2}\right)+g\left(x^{\prime}\right) .
$$

Firstly, we prove that $a$ is an extension of $g$. For this we distinguish two cases:

(i) If $x \in \operatorname{diad} D \cap\left[0, \frac{r}{2}\left[\right.\right.$. Then $x=0 \cdot \frac{r}{2}+x$, so

$$
a(x)=0 \cdot g\left(\frac{r}{2}\right)+g(x)=g(x) .
$$

(ii) If $x \in \operatorname{diad} D \cap\left[\frac{r}{2}\left[\right.\right.$. Then $x=1 \cdot \frac{r}{2}+x^{\prime}$, so

$$
a(x)=1 \cdot g\left(\frac{r}{2}\right)+g\left(x^{\prime}\right)=g\left(\frac{r}{2}+x^{\prime}\right)=g(x) .
$$

This entails $a$ is really an extension of $g$.

Secondly, we prove that $a$ is additive on $\operatorname{diad} D_{r}=\operatorname{diad} D$. Let $x, y \in$ $\operatorname{diad} D_{r}$ be arbitrary elements, and

$$
x=n_{x} \frac{r}{2}+x^{\prime}, \quad y=n_{y} \frac{r}{2}+y^{\prime} .
$$

We distinguish two cases again:

(i) If $x^{\prime}+y^{\prime} \in D_{\frac{r}{2}}$, then

$$
a(x+y)=\left(n_{x}+m_{y}\right) g\left(\frac{r}{2}\right)+g\left(x^{\prime}+y^{\prime}\right)=a(x)+a(y) .
$$

(ii) If $x^{\prime}+y^{\prime} \in\left[\frac{r}{2}, r\left[\cap D\right.\right.$, then $z^{\prime}+\frac{r}{2}=x^{\prime}+y^{\prime}$

$$
\begin{aligned}
a(x+y) & =\left(n_{x}+m_{y}+1\right) g\left(\frac{r}{2}\right)+g\left(z^{\prime}\right) \\
& =n_{x} g\left(\frac{r}{2}\right)+m_{y} g\left(\frac{r}{2}\right)+g\left(x^{\prime}\right)+g\left(y^{\prime}\right)=a(x)+a(y) .
\end{aligned}
$$


That is to say, $a$ is additive on the required set.

Thirdly, we extend $a$ onto the whole real line. It is clear that $\operatorname{diad} D$ with the usual addition is a subsemigroup of the additive group of real numbers. We can apply the theorem of Dhombres and Ger (see [9] or [11, Theorem 18.1.1.]). Which exactly says that there is a (not necessarily unique) homomorphism $A: \mathbb{R} \rightarrow \mathbb{R}$ such that $A_{\mid \operatorname{diad} D}=a$.

\section{Properties of Quasi-Arithmetic Expressions}

Let $I \subset \mathbb{R}$ be a proper interval, $\varphi: I \rightarrow \mathbb{R}$ be an invertible function such that its inverse has a dyadically closed domain. Then the following two place function

$$
A_{\varphi}(x, y):=\varphi^{-1}\left(\frac{\varphi(x)+\varphi(y)}{2}\right), \quad x, y \in I,
$$

is called a quasi-arithmetic expression generated by $\varphi$.

Besides of the required conditions, $A_{\varphi}$ is well-defined.

If $\varphi$ continuous and strictly monotone, then it fulfils the requirements above. The resulted set is the class of quasi-arithmetic means.

If $\varphi$ is an invertible additive function, then the corresponding quasiarithmetic expression is the arithmetic mean. If $\varphi=a \circ \log$, where $a$ is an invertible additive function again, we get the geometric mean. These phenomenon are called absorbing irregularity. In other words, a very regular expression can be generated by a very irregular one.

The following two observations shows that irregularity of the generating function is not always absorbed. As a consequence we have that the class of quasi-arithmetic expressions is really larger than the class of quasi-arithmetic means.

Observation 1. The class of quasi-arithmetic means are strictly contained by the class of quasi arithmetic expressions.

Proof. Let $I=\mathbb{R}$ and $a: \mathbb{R} \rightarrow \mathbb{R}$ be an arbitrary non-continuous, additive, self-bijection of the reals, and $\varphi=\exp \circ a$, then the quasi arithmetic expression generated by $\varphi$ is the following

$$
A_{\text {exp } \circ a}(x, y)=a^{-1} \circ \log \left(\frac{\exp (a(x))+\exp (a(y))}{2}\right), \quad x, y \in I .
$$

We prove that $A_{\varphi}$ is non-continuous, so it cannot be a quasi-arithmetic mean.

Assume that $A_{\varphi}$ is continuous, then the image of every connected set by $A_{\varphi}$ is connected.

$$
A_{\text {exp } \circ a}(\mathbb{R}, 0)=a^{-1} \circ \log \left(\frac{\exp (a(\mathbb{R}))+1}{2}\right)=a([-\log 2, \infty[)
$$

If the right hand side is connected, then it is an interval. Because $a$ is noncontinuous it is unbounded (from below and from above) on every non-empty 
interval (see e.g. [11]). So $a([-\log 2, \infty[)$ is an unbounded interval. The function $a$ is bijective, so it is surjective too, we have $a([-\log 2, \infty[) \neq \mathbb{R}$. This entails the existence of $b \in \mathbb{R}$ such that

$$
a([-\log 2, \infty[)=[b, \infty[, \text { or }] b, \infty[, \text { or }]-\infty, b], \text { or }]-\infty, b[.
$$

In all cases we get the boundedness of $a$ either from below or from above. It follows that $a$ is continuous, which is a contradiction.

The quasi-arithmetic means have the intern property, that is, their values are always between the minimum and the maximum of the variables. Intern property of the construction in the previous proof is an open problem, however, it is possible to construct a quasi-arithmetic expression which is non-continuous and it is not a mean.

Observation 2. There is a non-continuous quasi-arithmetic expression which is not a mean.

Proof. ${ }^{1}$ Let $\varphi: \mathbb{R} \rightarrow \mathbb{R}$ be the following function

$$
\varphi(x)= \begin{cases}x, & \text { if } x \in \mathbb{Q} \\ -x, & \text { if } x \in \mathbb{R} \backslash \mathbb{Q} .\end{cases}
$$

Then $\varphi=\varphi^{-1}$, and $A_{\varphi}(1, \sqrt{2})=\frac{\sqrt{2}-1}{2}<1<\sqrt{2}$. So, $A_{\varphi}$ is not a mean.

If $x_{n} \rightarrow \sqrt{2}$, and $x_{n} \in \mathbb{Q}$, we have

$$
A_{\varphi}\left(x_{n}, 1\right)=\frac{x_{n}+1}{2} \rightarrow \frac{\sqrt{2}+1}{2} \neq \frac{\sqrt{2}-1}{2}=A_{\varphi}(\sqrt{2}, 1)
$$

So, $A_{\varphi}$ is not continuous.

\section{Equality Problem of Quasi-Arithmetic Expressions}

Here we solve the following problem. Let $\varphi, \psi: I \rightarrow \mathbb{R}$ be invertible functions with inverses having dyadically closed domains. What is the sufficient and necessary condition of the equality of the generated quasi-arithmetic expressions? That is to say, solve the following functional equation!

$$
\varphi^{-1}\left(\frac{\varphi(x)+\varphi(y)}{2}\right)=\psi^{-1}\left(\frac{\psi(x)+\psi(y)}{2}\right), \quad x, y \in I .
$$

Theorem 2. Two quasi-arithmetic expressions generated by $\varphi$ and $\psi$ are equal on the interval $I$ if and only if there exists an invertible additive function $a: \mathbb{R} \rightarrow \mathbb{R}$ (not necessarily unique), and a real constant b such that

$$
\varphi(x)=a \circ \psi(x)+b, \quad x \in I .
$$

\footnotetext{
${ }^{1}$ Gyula Maksa's example, Oral communication.
} 
Proof. From the assumption $A_{\varphi}=A_{\psi}$ we have

$$
\psi \circ \varphi^{-1}\left(\frac{\varphi(x)+\varphi(y)}{2}\right)=\frac{\psi(x)+\psi(y)}{2}, \quad x, y \in I .
$$

Using the substitutions $\psi \circ \varphi^{-1}=: f: \operatorname{dom} \varphi^{-1} \rightarrow \mathbb{R}, \varphi(x)=: u, \varphi(y)=: v$, from the previous equation we get the following.

$$
f\left(\frac{u+v}{2}\right)=\frac{f(u)+f(v)}{2}, \quad u, v \in D=: \operatorname{dom} \varphi^{-1} .
$$

We can assume without losses that $0 \in D$. Substituting $v=0$ and $b:=f(0)$ into the equation above we have

$$
f\left(\frac{u}{2}\right)=\frac{f(u)+b}{2}, \quad u \in D
$$

Using (3) and (4) we get

$$
\frac{f(u)+f(v)}{2}=f\left(\frac{u+v}{2}\right)=\frac{f(u+v)+b}{2}, \quad(u, v) \in D_{\Delta} .
$$

Let $g: D \rightarrow \mathbb{R}, g(u):=f(u)-b$, then the previous equation entails the conditional additivity of $g$, that is to say,

$$
g(u+v)=g(u)+g(v), \quad(u, v) \in D_{\Delta} .
$$

Applying the extension theorem (Theorem 1.) for $g$, we have that there is and additive function $a: \mathbb{R} \rightarrow \mathbb{R}$ such that $a_{\mid D}=g$. Using this and the definitions of the functions $f$ and $g$, we have

$$
a(u)=f(u)-b=\psi \circ \varphi^{-1}(u)-b, \quad u \in D .
$$

This exactly means that

$$
a \circ \varphi(x)+b=\psi(x), \quad x \in I .
$$

So, the proof is ready.

\section{Open Problems}

Characterization of quasi-arithmetic means is well-known. The characterization of quasi-arithmetic expressions is open at this moment. For the formulation of the characterization theorem of quasi-arithmetic means we need the following conditions of a two place function $M: I \times I \rightarrow \mathbb{R}$ :

(i) Strict monotonicity: if $x<x^{\prime}$ then $M(x, y)<M\left(x^{\prime}, y\right)$ and the same for the second variable;

(ii) Continuity;

(iii) Bisymmetry: $M((x, y), M(u, v))=M(M(x, u), M(y, v))$;

(iv) Reflexivity: $M(x, x)=x$;

(v) Symmetry: $M(x, y)=M(y, x)$;

The next theorem due to Aczél can be found in [2]. 
Theorem 3 (Aczél, 1948). Conditions (i)-(v) are necessary and sufficient for the existence of a strictly increasing and continuous function $\varphi: I \rightarrow \mathbb{R}$ by which $M$ has the form

$$
M(x, y)=\varphi^{-1}\left(\frac{\varphi(x)+\varphi(y)}{2}\right), \quad x, y \in I .
$$

Conditions (iii)-(v) are fulfilled by quasi-arithmetic expressions too. The conditions (i) and (iv) implies intern property, that is, the value of the function is between the minimum and the maximum of the variables.

These ideas are motivate the following open problems:

Open problem 1. Is it possible to construct a non-continuous quasi-arithmetic expression which is a mean?

Open problem 2. Is it true that (iii)-(v) (maybe with additional conditions different from (i) and (ii)) characterize quasi-arithmetic expressions and (i) with (iii)-(v) characterize quasi-arithmetic expressions with intern property?

If the answer is affirmative for the first, so is for the second.

\section{Acknowledgements}

Open access funding provided by University of Debrecen (DE). This research is supported by the Hungarian Scientific Research Fund (OTKA) Grant NK 111651.

Open Access. This article is distributed under the terms of the Creative Commons Attribution 4.0 International License (http://creativecommons.org/licenses/ by/4.0/), which permits unrestricted use, distribution, and reproduction in any medium, provided you give appropriate credit to the original author(s) and the source, provide a link to the Creative Commons license, and indicate if changes were made.

\section{References}

[1] Aczél, J.: The notion of mean values. Norske Vid. Selsk. Forh., Trondhjem 19(23), 83-86 (1947)

[2] Aczél, J.: On mean values. Bull. Am. Math. Soc. 54, 392-400 (1948)

[3] Aczél, J., Skof, F.: Local Pexider and Cauchy equations. Aequationes Math. 73(3), 311-320 (2007)

[4] Burai, P., Dascăl, J.: The equality problem in the class of conjugate means. Aequationes Math. 84(1-2), 77-90 (2012)

[5] Daróczy, Z.: On the equality and comparison problem of a class of mean values. Aequationes Math. 81(3), 201-208 (2011)

[6] Daróczy, Z., Dascăl, J.: On the equality problem of conjugate means. Results Math. 58(1-2), 69-79 (2010) 
[7] Daróczy, Z., Losonczi, L.: Über die Erweiterung der auf einer Punktmenge additiven Funktionen. Publ. Math. Debrecen 14, 239-245 (1967)

[8] Daróczy, Z., Páles, Z.: Gauss-composition of means and the solution of the Matkowski-Sutô problem. Publ. Math. Debrecen 61(1-2), 157-218 (2002)

[9] Dhombres, J.G., Ger, R.: Conditional Cauchy equations. Glas. Mat. Ser. III 13(33)(1), 39-62 (1978)

[10] Gaál, M., Nagy, G.: Preserver problems related to quasi-arithmetic means of invertible positive operators. Integral Equ. Operator Theory, 90(1):Art. 7, 12, (2018)

[11] Kuczma, M.: An introduction to the theory of functional equations and inequalities. Birkhäuser, Basel, 2nd edn. Cauchy's equation and Jensen's inequality, Edited and with a preface by Attila Gilányi (2009)

[12] Lajkó, K., Mészáros, F.: Special cases of the generalized Hosszú equation on interval. Aequ. Math. 89(1), 71-81 (2015)

[13] Losonczi, L.: Equality of two variable weighted means: reduction to differential equations. Aequ. Math. 58(3), 223-241 (1999)

[14] Losonczi, L.: Equality of Cauchy mean values. Publ. Math. Debrecen 57(1-2), 217-230 (2000)

[15] Losonczi, L.: Equality of two variable Cauchy mean values. Aequ. Math. 65(1-2), 61-81 (2003)

[16] Losonczi, L.: Equality of two variable means revisited. Aequ. Math. 71(3), 228245 (2006)

[17] Losonczi, L., Páles, Z.: Equality of two-variable functional means generated by different measures. Aequ. Math. 81(1-2), 31-53 (2011)

[18] Makó, Z., Páles, Z.: On the equality of generalized quasi-arithmetic means. Publ. Math. Debrecen 72(3-4), 407-440 (2008)

[19] Maksa, G.: Solution of generalized bisymmetry type equations without surjectivity assumptions. Aequ. Math. 57(1), 50-74 (1999)

[20] Matkowski, J.: Generalized weighted and quasi-arithmetic means. Aequ. Math. 79(3), 203-212 (2010)

[21] Matkowski, J.: A functional equation related to an equality of means problem. Colloq. Math. 122(2), 289-298 (2011)

[22] Matkowski, J.: On homogeneous Lagrange means. Period. Math. Hungar. 68(2), 119-127 (2014)

[23] Páles, Z.: On the equality of quasi-arithmetic and Lagrangian means. J. Math. Anal. Appl. 382(1), 86-96 (2011)

[24] Páles, Z.: Extension theorems for functional equations with bisymmetric operations. Aequ. Math. 63(3), 266-291 (2002)

[25] Skof, F.: The general solution of the exponential Cauchy equation on a bounded restricted domain. Aequ. Math. 73(1-2), 144-155 (2007) 
Pál Burai

University of Debrecen

26 Kassai Road

Debrecen 4028

Hungary

e-mail: burai.pal@inf .unideb.hu

Received: January 4, 2019.

Accepted: June 27, 2019.

Publisher's Note Springer Nature remains neutral with regard to jurisdictional claims in published maps and institutional affiliations. 